\title{
Early Mesodermal Phenotypes in Splotch Suggest a Role for Pax3 in the Formation of Epithelial Somites
}

\author{
FRANK R. SCHUBERT, ${ }^{1}$ PATRICK TREMBLAY, ${ }^{2}$ AHMED MANSOURI, ${ }^{3}$ ANJA M. FAISST, ${ }^{3}$ \\ BIRGITTA KAMMANDEL, ${ }^{3}$ ANDREW LUMSDEN, ${ }^{1}$ PETER GRUSS, ${ }^{3}$ AND SUSANNE DIETRICH ${ }^{4 *}$ \\ ${ }^{1}$ King's College London, MRC Centre for Developmental Neurobiology, New Hunt's House, London, United Kingdom \\ ${ }^{2}$ Neurochem, Inc., St. Laurent, Quebec, Canada \\ ${ }^{3}$ Max-Planck-Institute for Biophysical Chemistry, Department of Molecular Cell Biology, Göttingen, Germany \\ ${ }^{4}$ King's College London, Department of Craniofacial Development, Guy's Tower, London, United Kingdom
}

\begin{abstract}
The paired box containing transcription factor Pax3 is a crucial regulator of dermomyotome and muscle development. However, the allelic series of Pax3/Splotch mutants also displays characteristic vertebral column malformations, which do not result from defective dorsoventral somite pattern. Rather, vertebral column and sclerotomal phenotypes are reminiscent of the phenotypes observed in the segmentation/somitogenesis mutants rachiterata and pudgy. Moreover, rostrocaudal somite pattern and somitic boundaries are disturbed in Splotch as monitored by the expression of Uncx4.1 and Lunatic fringe. Alterations in EphA4, Dll1, and Uncx4.1 expression are evident already in the condensing paraxial mesoderm, correlating with the first phase of Pax3 expression before and during somite formation. This finding suggests an early function of Pax3 during the formation of epithelial somites. $\odot 2001$ Wiley-Liss, Inc.
\end{abstract}

Key words: splotch; pudgy; rachiterata; Pax3; Pax1; EphA4; Dll1; lunatic fringe; Uncx4.1; somitogenesis

\section{INTRODUCTION}

In vertebrate embryos, the paraxial mesoderm is laid down during gastrulation as a loosely packed mesenchyme, embedded between neural tube/notochord, intermediate/lateral mesoderm, surface ectoderm and endoderm (reviewed by Christ and Ordahl, 1995; Gossler and Hrabě de Angelis, 1998; Pourquié, 1999). At a defined time after cells entered this mesodermal mesenchyme, they increase adhesion and form reiterated units or segments. This process is an inherited property of the paraxial mesoderm, as it occurs independently of environmental cues. The timing of segmentation is controlled by a molecular clock, for which the periodic expression of the basic helix-loop-helix transcription factor Hairy1 and the secreted glycosyltransferase Lunatic Fringe (homologues of the Drosophila segmentation factor Hairy and the boundary specific signalling molecule Fringe, respectively) are the first read-out. Cells periodically expressing those genes are synchronised by members of the Notch-Delta/Serrate signalling system for which Lunatic fringe is a modifier (del Barco Barrantes et al., 1999; Jiang et al., 2000; Moloney et al., 2000). A caudal to rostral gradient of maturation ensures that at the rostral end of the unsegmented paraxial mesoderm, the molecular clock is arrested. At this point, the rostrocaudal differences between somite halves and the segment boundaries are established, involving Notch-Dll/serrate signalling, Eph-Ephrin signalling, Presenilin transmembrane proteins and intrinsically, the activity of Mesp basic helixloop-helix transcription factors (reviewed by Pourquié, 1999).

In normal development, the segmenting paraxial mesoderm simultaneously epithelialises to form somites (reviewed by Gossler and Hrabě de Angelis, 1998; Pourquié, 1999). In amniotes, this process is controlled by the surface ectoderm, as ectoderm ablation prevents somitogenesis but not segmentation (Šošić et al., 1997; Palmeirim et al., 1998). In addition, the transcription factor Paraxis, which is crucial for epithelial somite but not segment formation, is induced by ectodermal signals and acts independent from Notch-Delta/Serrate signalling (Burgess et al., 1996; Barnes et al., 1997; Sošić et al., 1997; Johnson et al., 2001). How segmentation and somitogenesis are coordinated during development is not understood. Nevertheless, it is established that extrinsic cues continue to act on the somite to control its dorsoventral and mediolateral differentiation (reviewed by Christ and Ordahl, 1995; Gossler and Hrabě de Angelis, 1998), i.e., ectodermal signals together with signals from the dorsal neural tube maintain the epithelial organisation of the dorsal somite territory, which gives rise to the dermomyotome, the source of skeletal muscle and dorsal dermis. Ventrally, notochord and floor plate trigger the formation of the mesenchymal sclerotome, the source of vertebral column and ribs. Neural tube and notochord/

\footnotetext{
Grant sponsor: The Max-Planck Society; Grant sponsor: European Molecular Biology Organisation (EMBO); Grant sponsor: Human Frontier Science Program (HFSP).

*Correspondence to: Susanne Dietrich, King's College London, Department of Craniofacial Development, Floor 28 Guy's Tower, London, UK SE1 9RT. E-mail: susanne.dietrich@kcl.ac.uk

Received 16 May 2001; Accepted 17 August 2001
} 
floor plate cooperate medially to stimulate the formation of deep back muscles (epaxials) from the medial aspect of the dermomyotome, whereas ectoderm and intermediate/lateral mesoderm derived signals support the formation of all the laterally, ventrally, and superficially located muscles (hypaxials; reviewed by Dietrich, 1999).

While defects in dorsoventral and mediolateral somite pattern affect individual somitic derivates, defective segmentation and epithelial somite formation both lead to irregularly shaped and incompletely segregated blocks of paraxial mesoderm, with all derivatives showing poorly defined borders, irregular shape, and fusions (reviewed by Gossler and Hrabě de Angelis, 1998; Pourquié, 1999). For the sclerotome, this condition results in characteristic vertebral column malformations such as irregular and ectopic vertebral elements, half vertebrae, and block vertebrae (reviewed by Grüneberg, 1963; Töndury and Theiler, 1990). In extreme cases, the vertebral column displays a stiff array of misshapen cartilages and bones. Moreover, as the sclerotome dictates the position of the spinal nerves in the rostral sclerotome halves, the spinal nerves will be disorganised (reviewed by Christ and Ordahl, 1995; Gossler and Hrabě de Angelis, 1998; Pourquié, 1999). Several mouse mutants are known for their disorders of segmentation or somitogenesis (reviewed by Grüneberg, 1963; see also Mouse Locus Catalogue, Jackson Laboratory, Bar Harbor, ME). Likewise, disorganised somites are evident in several zebrafish mutants (van Eeden et al., 1996, 1998). However, the mutations map to different chromosomal loci of which only a few have been deciphered at the molecular level, suggesting that several genes govern the formation of segments and somites.

The vertebrate Pax3 gene is a member of the Pax multigene family of transcriptional regulators and harbours a paired box and a homeobox (reviewed by Chalepakis et al., 1993). The gene is mutated in the allelic series of Splotch mouse mutants (see also Mouse Locus Catalogue, Jackson Laboratory), which serve as model for human Waardenburg syndromes (I and III). In human patients and Splotch mice, Pax3 mutations are semidominant and homozygous lethal. The syndrome is tightly associated with structures that express Pax3 during embryogenesis such as the dorsal neural tube before neural tube closure, the emigrating neural crest cells, and the somitic dermomyotome (Goulding et al., 1991, 1994; Williams and Ordahl, 1994); in the absence of Pax3, the dorsal neural tube fails to close, leading to lumbosacral spina bifida aperta or craniorachischisis (Russell, 1947; Auerbach, 1954; reviewed by Chalepakis et al., 1993). Multiple neural crest deficiencies encompass reduction or absence of dorsal root and cranial ganglia; absence of melanocytes and Schwann cells; thymic, thyroid, and parathyroid defects; conotruncal heart defects (reviewed by Chalepakis et al., 1993); and malformation of neural crestderived bones such as the maxilla, the lacrimal and nasal bones, and the hyoid and thyroid cartilages (Tremblay et al., 1998). Loss of Pax3 in the dermomyotome results in reduced overall muscle masses, with appendicular, diaphragm, and ventrally located abdominal muscles missing altogether (reviewed by Dietrich, 1999). Interestingly, Pax3 mutations also lead to malformation of the vertebral column and ribs, which develop from the somitic sclerotome (Tremblay et al., 1998; Henderson et al., 1999; this study). As the sclerotome is devoid of Pax3 expression, the phenotype has been interpreted as secondary consequence of Pax3 lacking in the dermomyotome (Henderson et al., 1999). However, the paraxial mesoderm as a whole, including the prospective sclerotome, transiently expresses Pax3 before and during somite formation (Goulding et al., 1994; Williams and Ordahl, 1994; this study). Therefore, the Splotch vertebral column phenotype may result from a so far undetected early Pax3 function.

This study aims at clarifying the nature of the Splotch vertebral column defects and at completing the picture on Pax3 function in the paraxial mesoderm. We show that skeletal defects in Splotch arise from disorganised sclerotomes and encompass dorsolateral and ventromedial vertebral column components. Drosophila-type compensation experiments suggest that the sclerotomal disorganisation does not primarily result from defective dorsoventral somite pattern. Rather, the defects are intrinsic to the Splotch sclerotome and resemble the sclerotomal phenotypes found in the segmentation/somitogenesis mutants pudgy and rachiterata (Kusumi et al., 1998; see also Mouse Locus Catalogue, Jackson Laboratory). This similarity extends to perturbed rostrocaudal somite pattern and somitic boundaries in Splotch. Moreover, we found changes in the expression of Uncx4.1 (Mansouri et al., 1997; Neidhardt et al., 1997), EphA4 (formerly Sek1; Nieto et al., 1992), and Dll1 (Bettenhausen et al., 1995) in the condensing paraxial mesoderm, coinciding with the initial phase of Pax3 expression. On the other hand, the periodic expression of Lunatic fringe (Johnston et al., 1997; Forsberg et al., 1998; McGrew et al., 1998; Auhlela and Johnson, 1999) that is initiated before Pax3 expression was unaffected. Thus, Splotch vertebral column defects can be traced back to defects in the condensing paraxial mesoderm. This finding suggests that before its function in the dermomyotome, Pax3 plays a role during the formation of epithelially arranged somites.

\section{RESULTS \\ Expression of Pax 3 in the Unsegmented Paraxial Mesoderm}

The Pax3 gene is a crucial regulator of dermomyotome and muscle development (reviewed in Dietrich, 1999). However, earlier reports mentioned that chick Pax3 expression commences in the paraxial mesoderm before epithelial somites form, encompassing both the prospective dermomyotome and sclerotome (Goulding et al., 1994; Williams and Ordahl, 1994). Thus, there is 

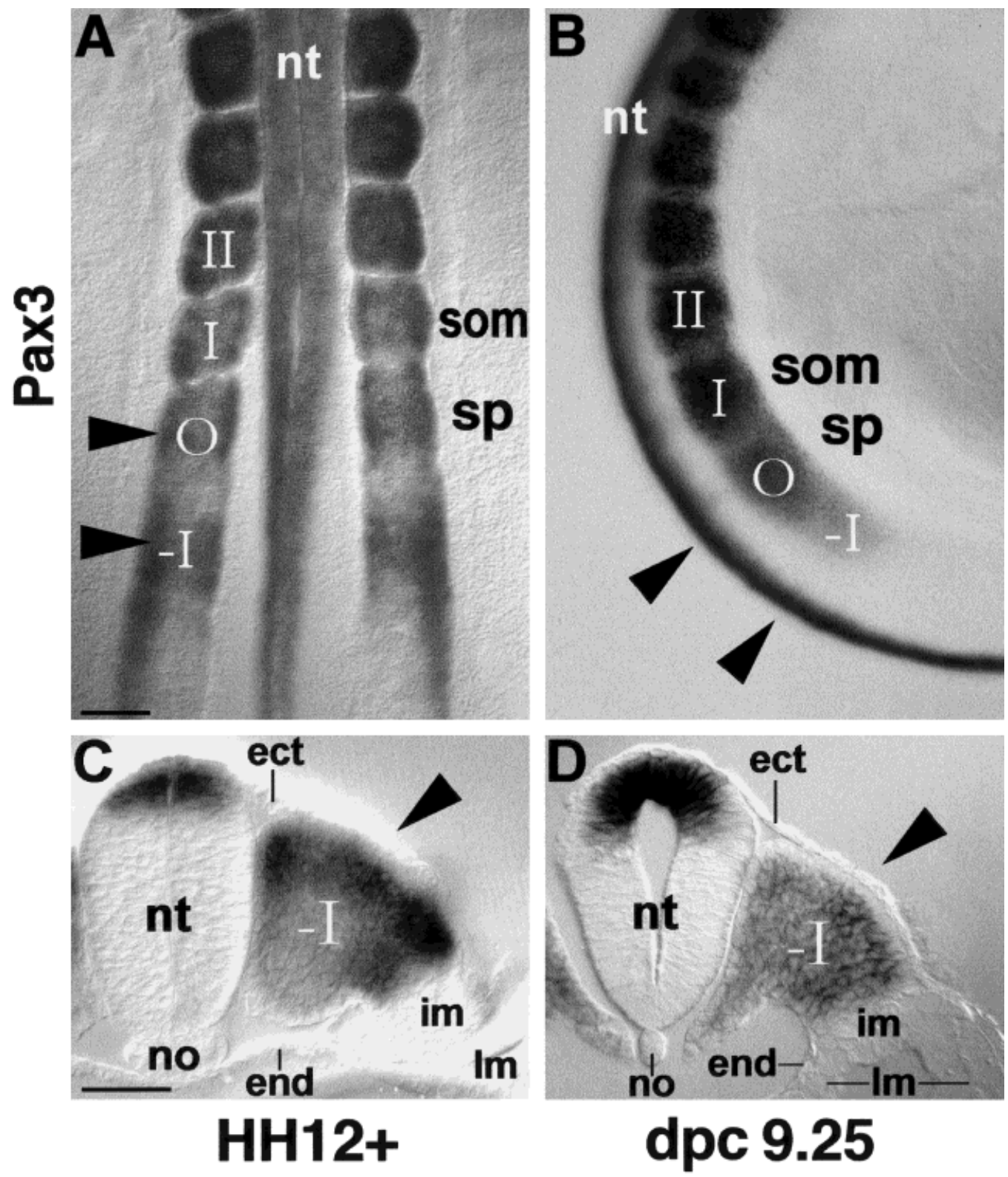

Fig. 1. Expression pattern of Pax3 during epithelial somite formation.

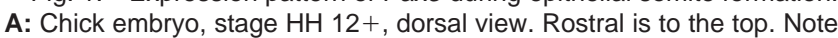
Pax3 signals in the newly formed somite (som) and in the rostral segmental plate (sp) in the next two somites to be formed (arrowheads). B: Mouse embryo at day post coitum (dpc) 9.25, lateral view. Rostral is to the top, dorsal to the left. As in the chick, Pax3 is expressed in the most recently formed somite (som) as well as in the next two prospective somites (arrowheads). C: Cross-section (right side) of the chick embryo in $A$ at the rostral end of the segmental plate, dorsal is to the top. Note Pax3 expression throughout the dorsoventral perimeter of the unseg- mented paraxial mesoderm. D: Cross-section (right side) of the mouse embryo in B, rostral end of the segmental plate, showing the same dorsoventral expression of Pax3 as seen for the chick. ect, surface ectoderm; end, endoderm; im, intermediate mesoderm; Im, lateral mesoderm; no, notochord; nt, neural tube; som, recently formed somite; sp, segmental plate/unsegmented paraxial mesoderm. Somites are numbered according to the nomenclature of Christ and Ordahl (1995), modified by Pourquié (1999). Scale bars $=100 \mu \mathrm{m}$ in A (applies to A,B), 50 $\mu \mathrm{m}$ in $\mathrm{C}$ (applies to C,D). a possibility for a so far unexplored, early function of Pax3 during initial stages of vertebrate somite formation. To investigate whether this possibility was realistic, we re-examined the expression pattern of Pax3 in chick and mouse embryos. In chick embryos at Hamburger and Hamilton stages $\mathrm{HH} 8-18$, Pax3 was ex- pressed at the rostral end of the unsegmented paraxial mesoderm (Fig. 1A). Expression was confined to one or two stripes, which traversed the entire dorsoventral perimeter of the tissue, thus prefiguring the next one or two somites to be formed (Fig. 1A,C, arrowheads). In mouse embryos at day 8-11 post coitum (dpc), a similar 
pattern was evident (Fig. 1B,D, arrowheads). This finding confirms that, before and during epithelial somite formation, the entire paraxial mesoderm passes though a phase of Pax3 expression.

\section{Comparative Analysis of Vertebral Column Phenotypes in Pax3/Splotch Mutants, Sclerotome, and Somitogenesis Mutants}

\section{Vertebral column phenotypes in the allelic se- ries of Splotch mutants}

Ossifying vertebral column. Upon epithelial somite formation, the developing amniote sclerotome rapidly turns off Pax3 (Goulding et al., 1994; Williams and Ordahl, 1994; Dietrich et al., 1997). Thus, had Pax3/ Splotch mouse mutants developed defects in the vertebral column, which is a sclerotomal derivative, this finding would point toward a functional relevance of the initial Pax3 expression. Therefore, we investigated skeletal phenotypes in the allelic series of Pax3/ Splotch mutants by using alcian blue-alizarin red staining (Kessel et al., 1990). We first examined dpc 16.5 and 18.5 skeletons, because at these stages, the ventromedial components (vertebral bodies and intervertebral discs) as well as the dorsolateral components (neural arches and ribs) of the axial skeleton are all preformed in cartilage (blue staining), and ossification is well under way (red staining). We concentrated on Splotch delayed $\left(S p^{d}\right)$ homozygotes and $S p^{d} / S p$ and $S p^{d} / S p 2 H$ compound heterozygotes, as these are the only Pax3 mutants developing to the required stages (reviewed by Chalepakis et al., 1993; see also Mouse Locus Catalogue, Jackson Laboratory). Wild-type animals and all types of Splotch heterozygotes displayed a regular array of vertebral column elements, with vertebral bodies separated by intervertebral discs (Fig. $2 \mathrm{~A}, \mathrm{v}, \mathrm{i}$ ). The neural arches were closing dorsally (not shown) with pedicles attached to the rostral portion of the vertebral bodies (Fig. 2A,p). Ribs were connected to the sternum, leading to regular subdivision into sternebrae (not shown). In $S p^{d}$ homozygotes and compound heterozygotes, however, at cervical and thoracic levels, individual neural arches or vertebral bodies were fused, in line with our earlier findings (Tremblay et al., 1998) and those of Henderson et al. (1999). Moreover, rib fusions were evident that always encompassed the first three ribs and that led to disorganisation of the sternum. The strongest phenotypes were observed in the lumbosacral region at the level of the open neural tube (Fig. 2B,C). Besides agenesis of the dorsal neural arches and spinous processes, multiple neural arch rudiments were fused (Fig. 2B,f). Vertebral bodies were fused also, with irregular and diminished intervertebral discs (Fig. 2C,I). The pedicles of the neural arches were weak and frequently attached to two consecutive vertebral bodies, such that vertebral bodies carried two neural arches (Fig. 2B,C, asterisks). Finally, superfluous cartilage condensations resided either between or were attached to vertebrae (Fig. 2B,C, arrowheads). Thus, in these mutants, both ventromedial and dorso- lateral vertebral column elements displayed mutant phenotypes. It is noteworthy that during the course of this study, we observed a single control animal displaying a spontaneous lumbosacral spina bifida. In the area of the open neural tube, the dorsal part of the neural arches was missing. However, as in human patients with spina bifida (Larsen, 1993), or in the spina bifida-affected mouse mutant curly tail (reviewed by Grüneberg, 1963; Töndury and Theiler, 1990; van Straaten and Copp, 2001), all other elements of the vertebral column were clearly separated and presented a normal morphology. This finding suggests that the fused and malformed vertebrae in $S p^{d}$ homozygotes and compound heterozygotes result directly from the impaired Pax3 function in the paraxial mesoderm rather than indirectly from the compromised neural tube.

Cartilaginous vertebral column at dpc 13.5. The $S p^{d}$ allele, harbouring a point mutation in the paired box of Pax3, is thought to be a hypomorph. To investigate whether Pax3 null mutations cause the same vertebral column phenotypes, we comparatively analysed the skeletons of Splotch delayed $\left(S p^{d}\right)$, Splotch $(S p)$, and Splotch $2 H\left(\mathrm{Sp}^{2 H}\right)$ homozygotes. The latter do not develop beyond dpc 13.5 and form a truncated Pax3 protein only, i.e., in $S p$, both the homeodomain and the transactivation domain are absent due to aberrant splicing, whereas in $S p^{2 H}$, a 32-bp deletion within the homeobox leads to premature translation termination that eliminates the transactivation domain (reviewed by Chalepakis et al., 1993; see also Mouse Locus Catalogue, Jackson Laboratory).

When analysed at dpc 13.5, the ventral and lateral parts of the vertebral column were well-defined cartilages in both controls and mutants. In wild-types and heterozygotes, vertebral bodies appeared compact and regularly spaced, carrying neural arches rostrally (Fig. 3A,E). By contrast, for all Splotch alleles, homozygotes and compound heterozygotes presented irregularly shaped and fused vertebral bodies (Fig. 3B,F, asterisks), fused ribs and fused neural arches (Fig. 3B,F,f). The lumbosacral phenotypes appeared even clearer than at dpc 16.5 or 18.5 (Fig. 3F) with multiple vertebral bodies being fused, together with rostral reductions and additional, caudal extensions (Fig. 3F, arrowheads). Pedicles were delicate (Fig. 3F,p) and frequently connected two adjacent vertebrae (Fig. 3B,F, asterisks). Finally, additional interconnecting cartilage condensations resided between the neural arch rudiments (Fig. 3F, arrowheads), resulting in a fused strip of cartilage (Fig. 3F,f). Thus, in all the Splotch alleles, the same skeletal defects develop that can be traced back to cartilaginous stages of vertebral column development.

Shape of individual cartilage condensations at dpc 12.5. Two possible scenarios may account for the Splotch skeletal phenotypes: either the cartilage condensations contributing to a vertebra are initially laid down properly but overgrow and fuse secondarily, or 

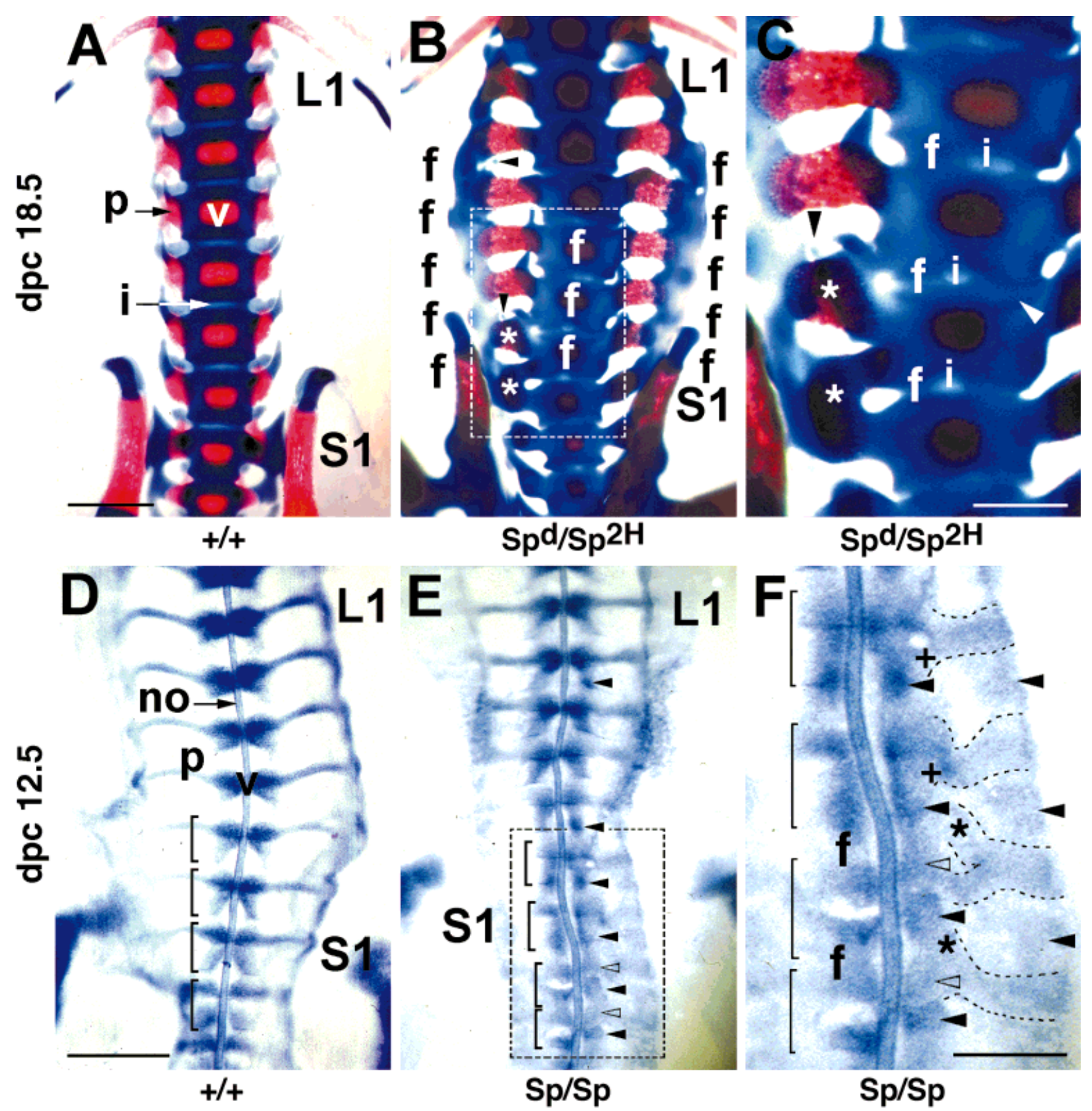

Fig. 2. Lumbosacral vertebral column of wild-type and Splotch mice at dpc $18.5(\mathbf{A}-\mathbf{C})$ and $12.5(\mathbf{D}-\mathbf{F})$, ventral views, rostral is to the top. A: Wild-type. The vertebral column is laid down in cartilage (blue staining). Ossification (red staining) is well under way both ventromedially in the vertebral bodies $(v)$ and dorsolaterally in the pedicles of the neural arches (p). Note the regular pattern of vertebrae with the pedicles of the neural arches attached to the rostral portion of the vertebral bodies. The vertebral bodies are spaced by cushion-like intervertebral discs (i). B: $S p^{d}$ / $S p^{2 H}$. Dorsolateral and ventromedial vertebral column components are malformed. The dorsal parts of the neural arches are absent due to lumbosacral rachischisis. In addition, the neural arch rudiments are fused (f). Several pedicles are connected to two consecutive vertebral bodies $\left.{ }^{*}\right)$. Ventromedially, intervertebral discs are reduced and several vertebral bodies are fused (f). Additional cartilaginous elements are found between or attached to individual vertebrae (arrowheads). C: Higher magnification of the boxed area in B. Note the irregular and fused vertebral bodies ( $f$ ), the reduced intervertebral discs (i), and the ectopic cartilage condensation (black arrowhead). Pedicles connected to two vertebrae are marked by asterisks. The white arrowhead marks a vertebral body that, at the anterior margin, shows the morphology of a partially duplicated, posterior element. D: Wild-type. The anlagen of the vertebral bodies and the pedicles of the neural arches are preformed in cartilage (blue staining). Note that the vertebral bodies are composed of several individual cartilage condensations. The rostral condensations in line with the neural arch anlagen are strongly stained, indicating more densely packed cells. They unite with the caudal elements as indicated by brackets. E: Sp/Sp. Note the prominent ventromedial phenotype: the cartilaginous elements of vertebral bodies are poorly demarcated (brackets), and some left and right halves are out of register. Additional cartilage elements are visible at the caudal border of vertebral bodies (arrowheads, indicated only on the right side), whereas rostral vertebral body elements are underdeveloped (open arrowheads). F: Higher magnification of the boxed area in E. Note the additional cartilage condensations ventromedially, at the caudal border of vertebral bodies, and laterally, between the neural arch rudiments (arrowheads, indicated only on the right side) which begin to fuse with the neighbouring vertebral elements (f). Some pedicles (outlined) are connected to two consecutive vertebral bodies $\left(^{*}\right)$ or to both the rostral and the caudal elements of the same vertebral body $(+)$. f, fused vertebral column element; i, intervertebral disc; L1, first lumbar vertebra; no, notochord; $p$, pedicle of the neural arch; $\mathrm{S} 1$, first sacral vertebra; $\mathrm{v}$, vertebral body. Scale bars $=1.75 \mathrm{~mm}$ in A (applies to A,B), $875 \mu \mathrm{m}$ in C, $650 \mu \mathrm{m}$ in $D$ (applies to $D, E), 325 \mu \mathrm{m}$ in F. 

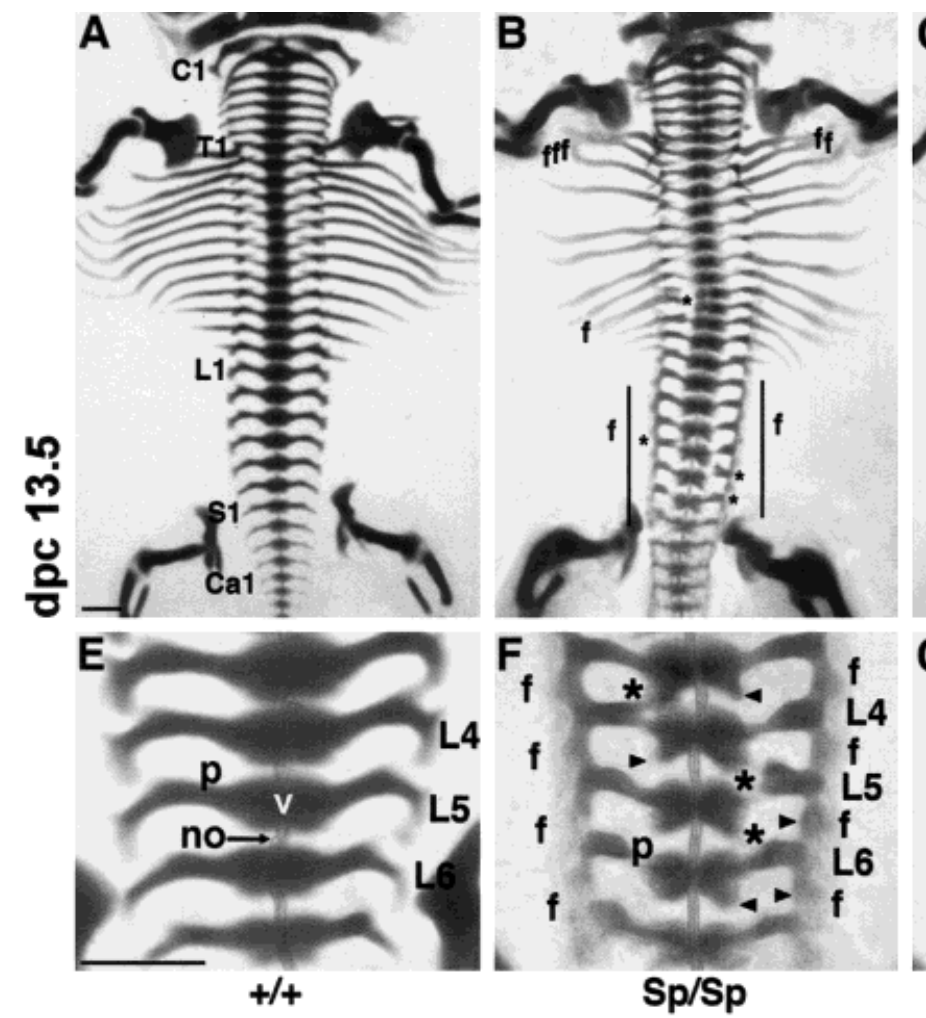
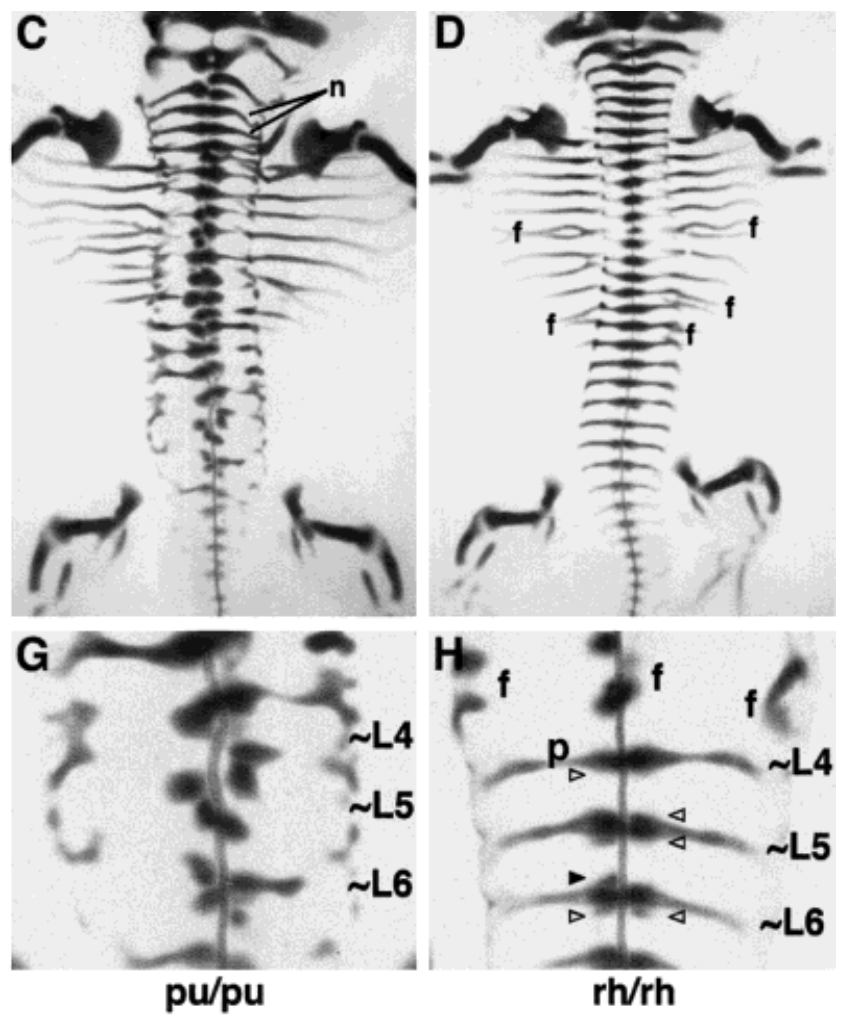

Fig. 3. Comparative vertebral column analysis of wild-type mice, Splotch and segmentation/somitogenesis mutants at dpc 13.5 (ventral views, rostral is to the top). In all animals, the ventromedial and lateral parts of the vertebral column as well as the appendicular skeleton are laid down in cartilage. E-H: Higher magnification of the lower lumbar vertebral column. A,E: Wild-type control. Note the regular array of vertebrae. B,F: Splotch. Note irregularly shaped vertebrae in the thoracic and lumbosacral regions. Ventromedial vertebral column components: Vertebral bodies are irregular and may carry cartilaginous extensions at the caudal border (arrowheads) that probably stem from the extra condensations visible at day post coitum (dpc) 12.5. Dorsal and lateral components: Rib fusions ( $f$ ) are evident that always encompass the first three ribs. Several pedicles are reduced $(p)$ or enlarged and connected to two consecutive vertebral bodies $\left({ }^{*}\right)$. Ectopic cartilage condensations (arrowheads) reside between the fusing neural arches (f). C,G: The segmentation mutant pudgy. All ventromedial and dorsolateral vertebral column components are highly disorganised. Vertebrae are represented by irregularly shaped cartilage condensations; individual vertebral bodies, arches, and ribs are missing or fused to their neighbours. Two normal vertebrae are indicated in the cervical region (n). D: The segmentation/somitogenesis mutant rachiterata. Most of the vertebral column forms normally. At irregular intervals, disproportionate, split, or fused vertebrae along with fused ribs develop. H: $r h / r h$ animal displaying a stronger lumbosacral phenotype. Note fused vertebral elements (f) absent or reduced pedicles ( $p$ ), reduced vertebral bodies (open arrowheads), and additional cartilaginous condensations (arrowheads). C1, first cervical vertebra; Ca1, first caudal vertebra; $n$, normal vertebra; $T 1$, first thoracic vertebra; others as before. Due to frequent vertebral fusion, only the approximate vertebral position could be indicated for $p u / p u(G, J)$ and $r h / r h(K)$. Scale bars $=650 \mu \mathrm{m}$ in $A$ (applies to A-D), in E (applies to $\mathrm{E}-\mathrm{H}$ ). they are improperly segregated in the first place. To discriminate between these possibilities, we examined the skeletal phenotypes of all Splotch alleles at dpc 12.5. At this stage, lumbosacral and tail vertebral bodies of control animals were butterfly-shaped and consisted of several individual cartilage condensations, revealing their two-somite origin (Fig. 2D): the more strongly stained, rostrally located cartilage elements were aligned with the pedicles of the neural arches, both developing from caudal somite halves. The more weakly labelled cartilage elements encompassed the caudal part of the vertebral bodies and originated from the rostral half of the next somite (reviewed by Gossler and Hrabě de Angelis, 1998). In all combinations of homozygous and compound heterozygous Splotch alleles, the anlagen of vertebral bodies were poorly de- marcated, irregular in size, and several left and right sides were out of register (Fig. 2E,F, brackets). Moreover, the individual components of various vertebrae were malformed: while cartilaginous elements in the rostral part of the vertebral bodies appeared underdeveloped as they stained only weakly (Fig. 2E,F, open arrowheads), additional centres of enhanced cartilage formation were evident at the caudal border (Fig. 2E,F, arrowheads, indicated on the right side only). Frequently, these cartilage condensations were continuous with the next vertebral body anlage (Fig. $2 \mathrm{~F}$,f). The anlagen of the neural arches were poorly defined and consisted of broad cartilage condensations with reduced pedicles. For several vertebrae, pedicles were connected to both the rostral part of one vertebral body and the caudal part of the previous vertebral body, 
such that vertebral bodies were again connected to two neural arches (Fig. 2F, asterisks). Neural arches attached to both the rostral and the caudal part of the same vertebral body were also observed (Fig. $2 \mathrm{~F}$, crosses). Finally, the ectopic condensations we had observed between the neural arches at dpc 13.5, were already clearly visible as individual cartilage elements (Fig. 2F, arrowheads). These findings suggest that the Splotch vertebral column phenotypes arise from poorly segregated, out of register, and ectopic cartilage condensations.

Vertebral column phenotypes of Pax1/undulated extensive and Pax3/Splotch double homozygotes. Henderson and colleagues (1999) suggested that the Splotch vertebral column defects are caused by a defective interaction between sclerotome and overlying dermomyotome/myotome, as a consequence of Pax3 lacking in the dermomyotome. Although this scenario may account for the misshapen dorsal and lateral aspects of the vertebral column, it is unlikely to cause poorly segregated, out of register, and ectopic cartilage condensations ventromedially. Nevertheless, it cannot be excluded that the enhanced apoptosis within the Pax3-deficient, hypotrophied dermomyotomes (Borycki et al., 1999) may permit the development of hypertrophied sclerotomes, thereby indirectly causing ventromedial vertebral column defects. In such case, mutants that lack vertebral elements due to underdeveloped sclerotomes such as the Pax1 null mutant undulated extensive (un ${ }^{e x}$; Wallin et al., 1994; Dietrich and Gruss, 1995; Mouse Locus Catalogue, Jackson Laboratory; Fig. 3B,E) would be expected to compensate the Splotch phenotype in double homozygotes. Therefore, we performed a Drosophila-type compensation experiment, crossing Splotch heterozygotes with $u n^{e x} / u n^{e x}$ homozygotes. $S p u n^{e x} /+u n^{e x}$ were viable with $u n^{e x}$ specific vertebral column phenotypes and $S p$-specific white spotting. Double homozygotes did not develop beyond dpc 13.5 and display a superposition of $u n^{e x}$ and Splotch phenotypes such as reduction or dysgenesis of vertebral bodies, vertebral and rib fusions, and additional cartilaginous elements (data not shown). This finding suggests that Splotch and undulated extensive phenotypes do not compensate each other. Thus, Splotch vertebral column defects may arise from a primary defect within the sclerotome rather than indirectly, from a defective dermomyotome/myotome.

Vertebral column phenotypes of Dll3/pudgy and rachiterata mice. The various homozygous and compound heterozygous Splotch mutants all show fused ribs along with malformed and fused vertebrae and ectopic cartilages, which are common features of mice deficient in mesoderm segmentation or somite epithelialisation. Thus, consistent with the intrinsic nature of the Splotch vertebral column defects as well as the early phases of Pax3 expression, Pax3 mutations may disturb segmentation/somitogenesis. Therefore, we needed to establish the degree of similarity between vertebral column phenotypes seen in Splotch and in known segmentation/somitogenesis mutants. We chose pudgy (pu/pu) and rachiterata ( $r h / r h)$ mice, because they represent strong and weak segmentation/somitogenesis phenotypes, respectively (Mouse Locus Catalogue, Jackson Laboratory; Kusumi et al., 1998): in the Dll3 mutant pudgy (pu), mesoderm segmentation is defective in dpc $13.5 \mathrm{pu} / \mathrm{pu}$ mutants resulting in a highly disorganised vertebral column with multiple fused vertebrae and ribs, half vertebrae, block vertebrae or cartilaginous structures of unclear identity (Fig. 3C,G). This phenotype affected all axial levels and differed from the Splotch phenotype quantitatively as it was more severe. In $r h / r h$, fused somites are scattered along the rostrocaudal axis. Consequently, although several vertebrae develop normally, individual cartilage elements at dpc 13.5 were reduced, added, lacking, or fused (Fig. 3D,H), thus resembling the phenotype of Splotch. Different from Splotch, $r h / r h$ mice displayed homeotic transformations of cervical vertebrae and their lumbosacral phenotype was less severe.

\section{Comparative Analysis of the Sclerotomal Organisation in Pax3/Splotch, Dll3/pudgy, and rachiterata mice}

The qualitative similarities of vertebral column phenotypes in Splotch and the segmentation/somitogenesis mutants, in particular rachiterata, suggested that Pax3 may indeed play a role during early somite formation. On the other hand, in addition to the different severity of phenotypes, each of the mutants showed specific traits not shared by the others. Thus, it was important to directly assess the organisation of $S p$, $p u$, and $r h$ sclerotomes, by using Pax1 as a marker (Deutsch et al., 1988; Dietrich and Gruss, 1995). At dpc 12.0, this marker allows the alignment of sclerotomal phenotypes and phenotypes of the cartilaginous vertebral column; in the wild-type (Fig. 4A), the strongest Pax1 signals reside in the caudal parts of the lumbar sclerotomes and rostrally below the spinal nerves (sn). These areas show high rates of cell proliferation and contribute to the neural arches and the intervertebral discs and vertebral borders, respectively (Wilting et al., 1994, reviewed by Gossler and Hrabě de Angelis, 1998). Ventromedially, the areas of weaker Pax1 expression coincide with the cartilage forming vertebral body components, which are still separated by the initial intersegmental boundaries (arrowheads). The rostrocaudal differentiation of the sclerotome commences at dpc 10.5 (Fig. 4E), when elevated levels of Pax1 expression are confined to caudal sclerotome halves and to the rostral sclerotomal borders, whereas newly formed sclerotomes evenly express Pax1 throughout (not shown).

Different from the wild-type, all mutants showed irregular pattern of Pax1 up-regulation at dpc 12.0 and 10.5 , in line with their degree of vertebral disorganisation. Most prominent in pu/pu (Fig. 4B,F), Pax1 signals were highly disorganised and fused (f), as were the spinal nerves. Disorganisation and fusion of Pax1 domains was also detected in $r h / r h$ but was less common 

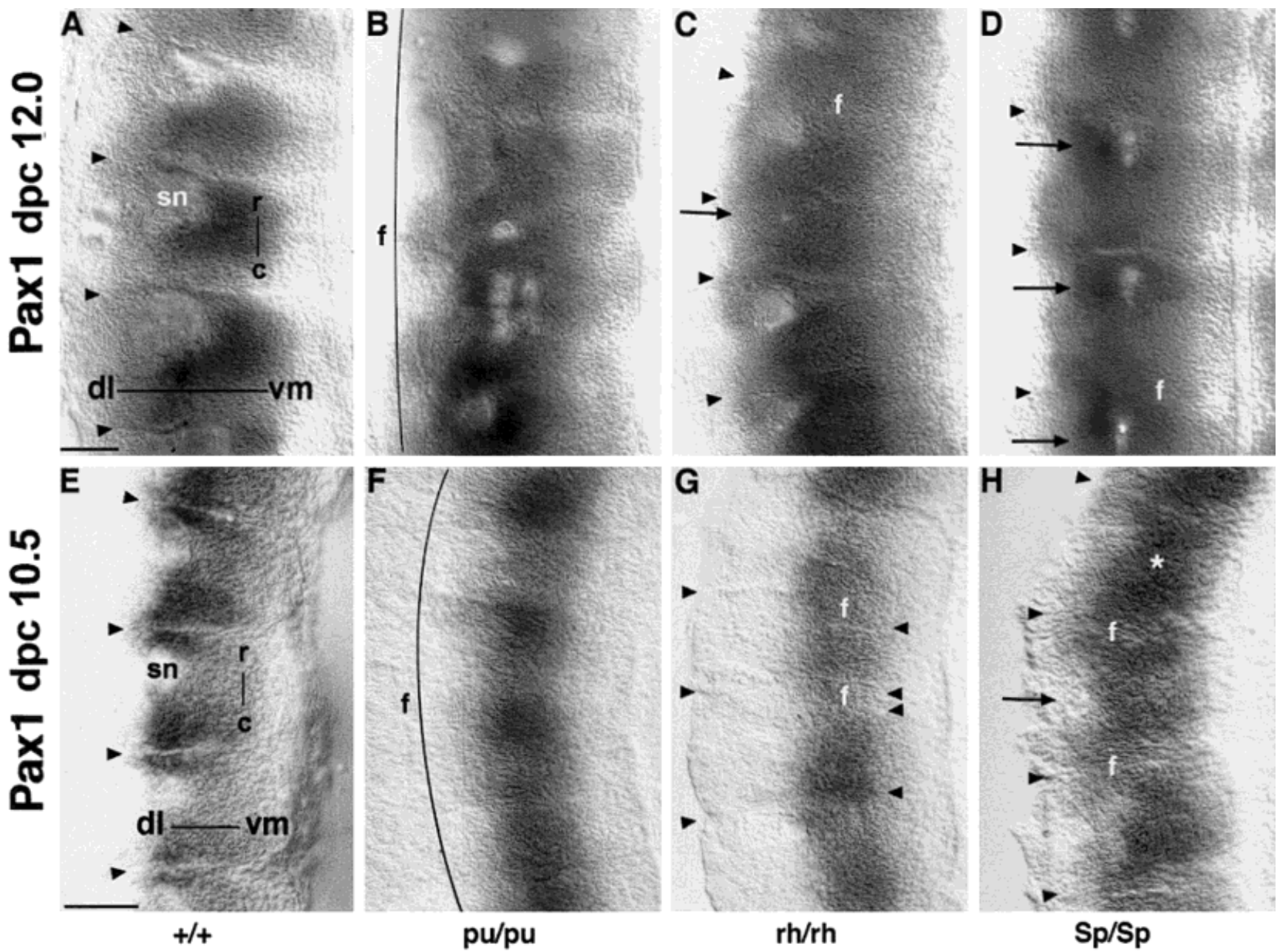

Fig. 4. Pax1 expression in differentiating somites of wild-type mice, segmentation/somitogenesis and Splotch mutants. A-D: Lumbar region, day post coitum (dpc) 12.0. View onto the medial aspect of the sclerotome after neural tube removal; rostral is to the top, dorsolateral to the left. A: Wild-type. The sclerotomes are separated by intersegmental fissures (position indicated by arrowheads). Note the strong Pax1 staining in the caudal sclerotome halves as well as in the centres of the sclerotomes. The spinal nerves pass through the lateral aspect of the rostral sclerotome halves (sn). B: pudgy. Pax1 signals are fused (f). Note the irregular array of elevated Pax1 signals and spinal nerves. C: rachiterata. Fused (f) and irregular sclerotomes with a reduced spinal nerve (arrow) are indicated. D: Splotch. The ventromedial to dorsolateral extent of Pax1 expression is normal. However, the three sclerotomes indicated (arrows) display irregular and fused (f) Pax1 signals with altered rostrocaudal pattern. Note the strong Pax1 signals in the rostral sclerotome halves, lateral to the Pax1-negative spots, which indicate the position of the motor axons (lumbosacral dorsal root ganglia are missing). In the caudal sclerotome halves and ventromedially, levels of Pax1 expression are reduced. E-H: Thoracic region, dpc 10.5. View as in A-D. E: Wild-

(Fig. 4C,G). In Splotch, the dorsoventral distribution of Pax1 signals was normal, underlining that the sclerotomes were not hypertrophied (data not shown). Similar to $\mathrm{pu} / \mathrm{pu}$ and $\mathrm{rh} / \mathrm{rh}$, Splotch sclerotomes exhibited fused Pax1 signals and an erroneous pattern of Pax1 up-regulation (Fig. 4D,H). At dpc 12.0, ectopic domains of elevated Pax1 expression were evident lumbosa-

type. The sclerotomes are clearly demarcated (position of the intersegmental fissures indicated by arrowheads). The highest level of Pax1 expression is found in the caudal sclerotome halves, destined to give rise to the rostral parts of the vertebral bodies and to the neural arches. In the lateral territory of the rostral sclerotome half, the Pax1-negative area corresponds to the developing spinal nerve. F: pudgy. All Pax1 signals are fused (f). Lighter and darker Pax1 staining appears at irregular intervals indicating incompletely segregated somites and disturbed rostrocaudal pattern. G: rachiterata. Three irregularly shaped and fused sclerotomes are evident with ill-defined rostrocaudal Pax1 pattern. H: Splotch. As at dpc 12.0, the rostrocaudal pattern, not the ventromedial to dorsolateral extent, of Pax1 expression is altered. The arrow indicates a sclerotome with two equally intense Pax1 signals in both halves, fused with the adjacent segments (f). The rostrally located sclerotome evenly expresses Pax 1 throughout $\left(^{*}\right)$. The approximate borders of the fused sclerotomes are indicated by arrowheads. c, caudal sclerotome half; dl, dorsolateral territory; vm, ventromedial territory; $r$, rostral sclerotome half; sn, spinal nerve; others as before. Scale bar $=400 \mu \mathrm{m}$ in A,E.

crally in the rostral sclerotome halves (Fig. 4D, arrows), adjacent to the Pax1-negative domains indicative of motor axons (dorsal root ganglia are reduced or absent due to neural crest deficiency; reviewed by Chalepakis et al., 1993; Tremblay et al., 1995). The position of these signals corresponded to that of the additional cartilage elements between the neural 

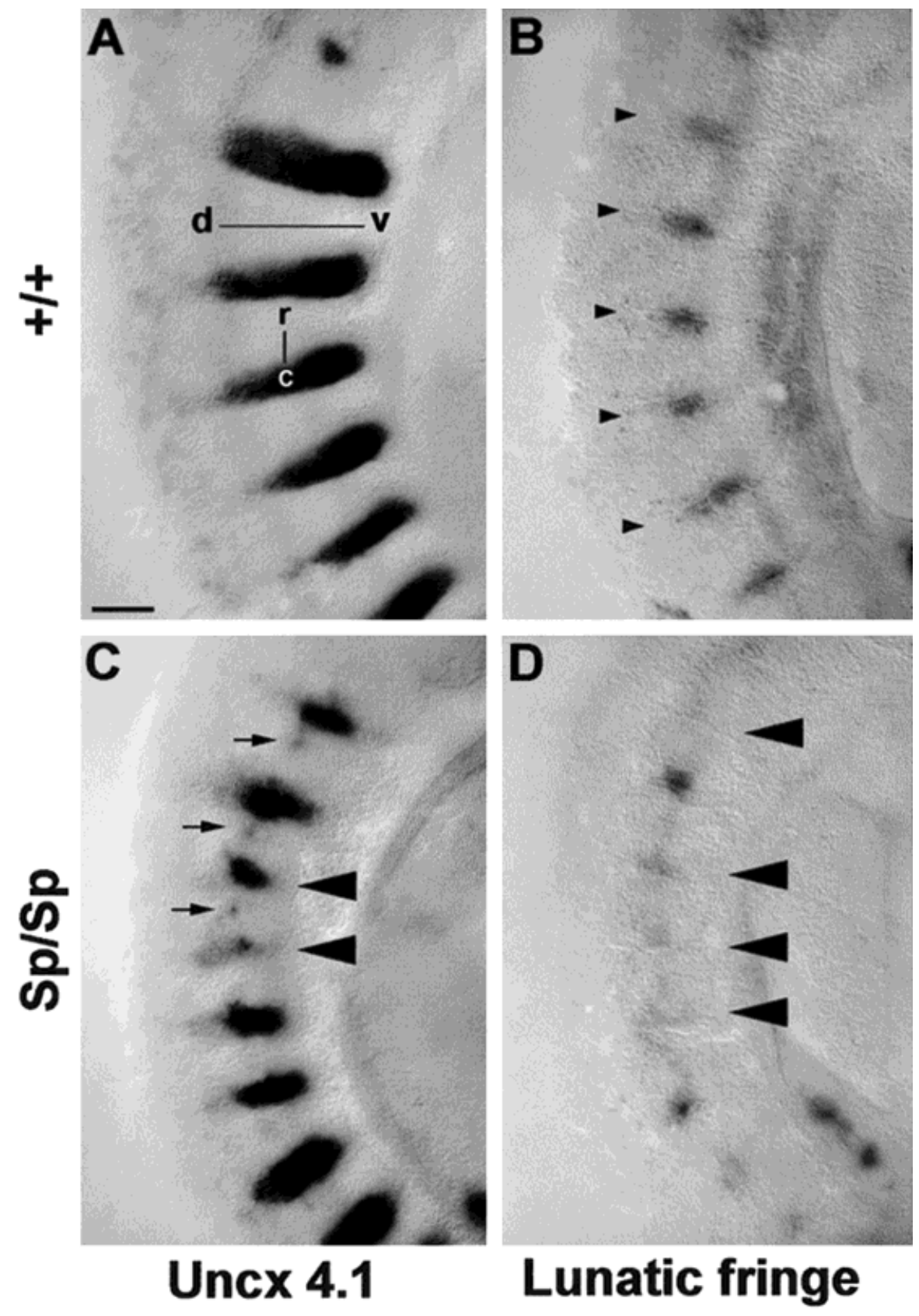

Fig. 5. Expression of Uncx4.1 and Lunatic fringe in differentiating somites of wild-type $(\mathrm{A}, \mathrm{B})$ and Splotch $(\mathrm{C}, \mathrm{D})$ embryos at day post coitum (dpc) 10.5. Lateral views, rostral to the top and dorsal to left; the neural tube has been removed. A,C: Expression of Uncx4.1 in cervical somites. A: Note the clearly demarcated, regularly spaced signals in the caudal halves of the wild-type sclerotomes. C: Irregular (arrowheads) and ectopic (arrows) signals are typical for Splotch, indicating disturbed rostrocaudal somite pattern. (B,D) Lunatic fringe expression in somites at forelimb levels. B: In the wild-type, Lunatic fringe sharply labels the somitic borders (small arrowheads). D: In Splotch, the signals are reduced and poorly defined (large arrowheads), indicating perturbed somitic boundaries. Abbreviations as before. Scale bar $=100 \mu \mathrm{m}$ in A (applies to A-D).

arches and at the caudal border of vertebral bodies. For all mutants, sclerotomal phenotypes could be traced back to the early, undifferentiated sclerotomes in dpc 10.5 tails, where Pax 1 signals were irregular and fused (not shown).

\section{Analysis of Rostrocaudal Somite Organisation and Somitic Boundaries in Splotch}

The similarity of sclerotomal phenotypes in $\mathrm{pu} / \mathrm{pu}$, $r h / r h$, and Splotch further supported the idea of Pax3 acting during early somite formation. If this were true, we would expect to find in Splotch (1) further evidence for disorganised rostrocaudal somite halves, besides the disturbed pattern of Pax1 up-regulation; and (2) disorganised somitic boundaries. Therefore, we investigated the cervicothoracic dpc 10.5 expression pattern of Uncx 4.1 and Lunatic fringe. The homeobox gene Uncx4.1 in the wild-type labels the caudal half-somites and subsequently the caudal sclerotome halves (Mansouri et al., 1997; Neidhardt et al., 1997; Fig. 5A). In Splotch (Fig. 5C), Uncx4.1 expression was disorganised, displaying irregular (arrowheads) and ectopic (arrows) sclerotomal signals. The secreted modulator of Notch-Delta/Serrate signalling, Lunatic fringe, ini- 
tially contributes to the timed formation of mesodermal segments as waves of expression sweep through the unsegmented paraxial mesoderm (Johnston et al., 1997; Forsberg et al., 1998; McGrew et al., 1998; Auhlela and Johnson, 1999). Expression resolves into caudally located stripes, and in the thorax of dpc 10.5 embryos, was confined to the somite borders (Fig. 5B). This pattern was disturbed in Splotch, as Lunatic fringe signals were irregular or missing (Fig. 5D, arrowheads).

\section{Analysis of Epithelialising Somites and the Unsegmented Paraxial Mesoderm in Splotch}

The alterations in Uncx4.1 and Lunatic fringe expression in differentiated somites at dpc 10.5 underlined that generalised somitic defects are present in Splotch, consistent with a role of Pax3 during early somite formation. To prove this hypothesis, we needed to trace the somitic phenotypes in Splotch back to the developing epithelial somite and the unsegmented paraxial mesoderm. Therefore, we analysed the initial expression of EphA4, Uncx4.1, Dll1, and Lunatic fringe in dpc 10,5 (Figs. 6, 7A,B) and dpc 12.0 (Fig. 7C,D) tails. The tyrosine kinase receptor EphA4 (formerly Sek1) is required for the formation of segmental borders in the paraxial mesoderm; dpc 10.5 wild-type mice, in addition to the tail bud, show two clearly defined stripes in the region where paraxial mesoderm is condensing to form somites (Fig. 6A; Nieto et al., 1992; Durbin et al., 1998). The rostral stripe was narrow and corresponded to the rostral half of the recently formed somite. The caudal stripe was broader and encompassed the next somite to be formed. The two stripes were separated by a clearly defined demarcation. In Splotch, EphA4 expression was disturbed (Fig. $6 \mathrm{D}$, arrowheads). The signals were weaker and poorly demarcated, suggesting that the compaction of the paraxial mesoderm and the segregation into orderly somites was perturbed. Comparing the Splotch and pudgy phenotypes, we found in the latter EphA4 staining at the correct distance from the tail bud, suggesting that the timing of expression was normal. However, in $p u / p u$ signals were confined to a rather fuzzy, irregularly shaped, and poorly demarcated domain rather than in stripes, underlining the strong segmentation phenotype (Fig. 6B). In rachiterata, EphA4 showed a wild-type-like expression pattern (Fig. 6C), reflecting that for many somites segmentation proceeds normally.

Early Uncx4.1 expression in the wild-type commences in the caudal half of each somite, briefly before epithelialisation is complete (Mansouri et al., 1997; Neidhardt et al., 1997; Fig. 7A,Ai). By contrast, the recently formed somites of Splotch show irregular Uncx4.1 signals with ill-defined borders (Fig. 7B,Bi, arrowheads). The Notch ligand Dll1 is expressed throughout the unsegmented paraxial mesoderm and, when the somite prepares for segregation at the level of somite -I, becomes restricted to the caudal halves (Bet- tenhausen et al., 1995; Fig. 7C). In Splotch (Fig. 7D), the Dll1 expression pattern in the unsegmented paraxial mesoderm was unchanged. At the level of somite -I, however, Dll1 expression retracted to the prospective caudal somite half along an irregular line (Fig. 7D, arrows), and the recently formed somites showed irregular or fused Dll1 signals (Fig. 7D, arrowheads). Finally, when we investigated the periodic expression of Lunatic fringe in the unsegmented paraxial mesoderm (Johnston et al., 1997; Forsberg et al., 1998; McGrew et al., 1998; Auhlela and Johnson, 1999), we did not detect any differences between wild-type and Splotch (not shown). Thus, although the molecular clock driving the segmentation of the paraxial mesoderm works normally in the absence of Pax3, the process of mesoderm compaction and epithelial somite formation at the rostral end of the unsegmented paraxial mesoderm is affected, in line with the onset of Pax3 expression. This finding suggests that, in the wild-type, Pax3 acts in the control of epithelial somite formation.

\section{DISCUSSION}

The paired and homeobox containing transcription factor Pax3 is an important regulator of embryonic development. The gene is mutated in the allelic series of Splotch mouse mutants, which show developmental defects at many sites of Pax3 expression (reviewed by Chalepakis et al., 1993; Mouse Locus Catalogue, Jackson Laboratory). Recent studies indicated that Splotch mice also develop vertebral column defects, although Pax3 is never expressed in the somitic sclerotome (Goulding et al., 1991, 1994; Williams and Ordahl, 1994; Tremblay et al., 1998; Henderson et al., 1999). On the other hand, no function has been associated so far with the expression of Pax3 in the unsegmented paraxial mesoderm, which encompasses also the prospective sclerotome. Providing a full account on the vertebral column phenotypes of three different Splotch alleles, we are able to show that, in the absence of Pax3, generalised vertebral column defects develop that are laid down by disorganised sclerotomes. These defects resemble defects found in the segmentation/ somitogenesis mutants pudgy and rachiterata (Mouse Locus Catalogue, Jackson Laboratory; Kusumi et al., 1998) and can be traced back to early defects within the condensing, unsegmented paraxial mesoderm. This finding suggests a requirement of Pax3 during mesoderm compaction and epithelial somite formation.

\section{Pax3 Mutants Develop Generalised Vertebral Column Defects}

Splotch mice develop well-known vertebral column defects in the area of the open neural tube, which include agenesis of the dorsal parts of the neural arches and of the spinous processes (Auerbach, 1954; Tremblay et al., 1998; Henderson et al., 1999). In addition, we found the rudiments of the neural arch laminae fused into a continuous cartilaginous mass, whereas numerous pedicles of the neural arches were 


\section{EphA4}
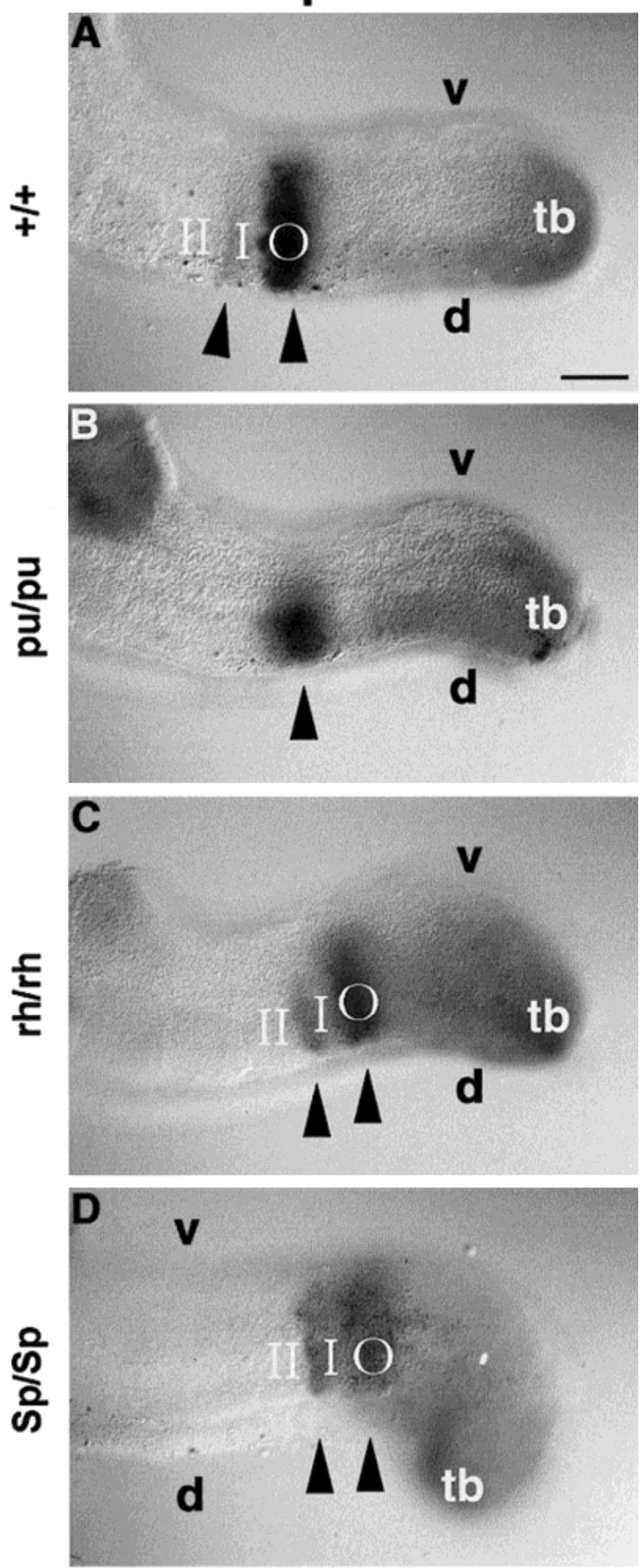

EphA4 erroneously connected to two consecutive vertebral bodies. Furthermore, ectopic cartilage elements were present, and vertebral bodies were fused. However, these vertebral malformations, although less pronounced, were also present at axial levels not affected by rachischisis. Moreover, in human patients and mice suffering from rachischisis, for instance the mouse mutant curly tail, although the neural arches fail to close and spinous processes are absent, the remaining vertebral components remain unchanged (reviewed by Grüneberg, 1963; Töndury and Theiler, 1990; Larsen, 1993; van Straaten and Copp, 2001; see also Mouse Locus Catalogue, Jackson Laboratory). Therefore, the irregular and fused vertebral bodies, neural arches, and ribs seen in the allelic series of Splotch mutants constitute a generalised vertebral column phenotype that randomly affects all axial levels.

\section{The Skeletal Malformations in Splotch Are Preformed at Cartilaginous and Mesenchymal Stages of Vertebral Column Development}

All aspects of the skeletal phenotype in Splotch are readily visible at cartilaginous stages of vertebral column formation at dpc 13.5. At dpc 12.5, when the individual components contributing to a vertebra are discernible lumbosacrally, we found reduced, malformed, or ill-defined anlagen for the vertebral bodies; pedicle anlagen attached to two neighbouring vertebral bodies; and ectopic cartilage condensations that interconnected adjacent vertebral bodies or neural arch rudiments. Moreover, several left and right halves of vertebrae appeared out of register. This finding suggested that the mesenchymal precursors of vertebrae, rather than the process of cartilage and bone formation are affected in Splotch. Indeed, by using Pax1 as marker (Deutsch et al., 1988; Dietrich and Gruss, 1995), we were able to detect a substantial disorganisation of the Splotch sclerotome: at dpc 12.0, ectopic up-regulation of $\operatorname{Pax} 1$ was evident in the rostral sclerotome halves, anticipating the position of the supernumerary cartilages observed later. At dpc 10.5, thoracic Pax1 signals were irregular and fused, the array of weaker and stronger signals within a segment was

Fig. 6. EphA4 expression during epithelial somite formation in wildtype mice, segmentation/somitogenesis and Splotch mutants at day post coitum (dpc) 10.5 (lateral view on tail, rostral is to the left, dorsal to the bottom). A: Wild-type. EphA4 is expressed in the tail bud and at the rostral end of the unsegmented paraxial mesoderm. Here, the signals are confined to a stripe at the rostral end of the recently formed somite and a broader stripe encompassing the next, epithelialising somite (arrowheads). B: pudgy. Note EphA4 expression in the paraxial mesoderm at the correct distance from the tail bud. The signal occupies a rostrocaudally broadened, but dorsoventrally shortened domain with poorly defined borders (arrowhead). C: rachiterata. Wild-type-like expression pattern of EphA4 (arrowheads). D: Splotch. EphA4 is expressed in broad, poorly demarcated stripes of almost equal intensity (arrowheads). $d$, dorsal; tb, tail bud; $v$, ventral; somite numbers as in Figure 1. Scale bar $=100 \mu \mathrm{m}$ in A (applies to A-D). 


\section{Uncx 4.1}
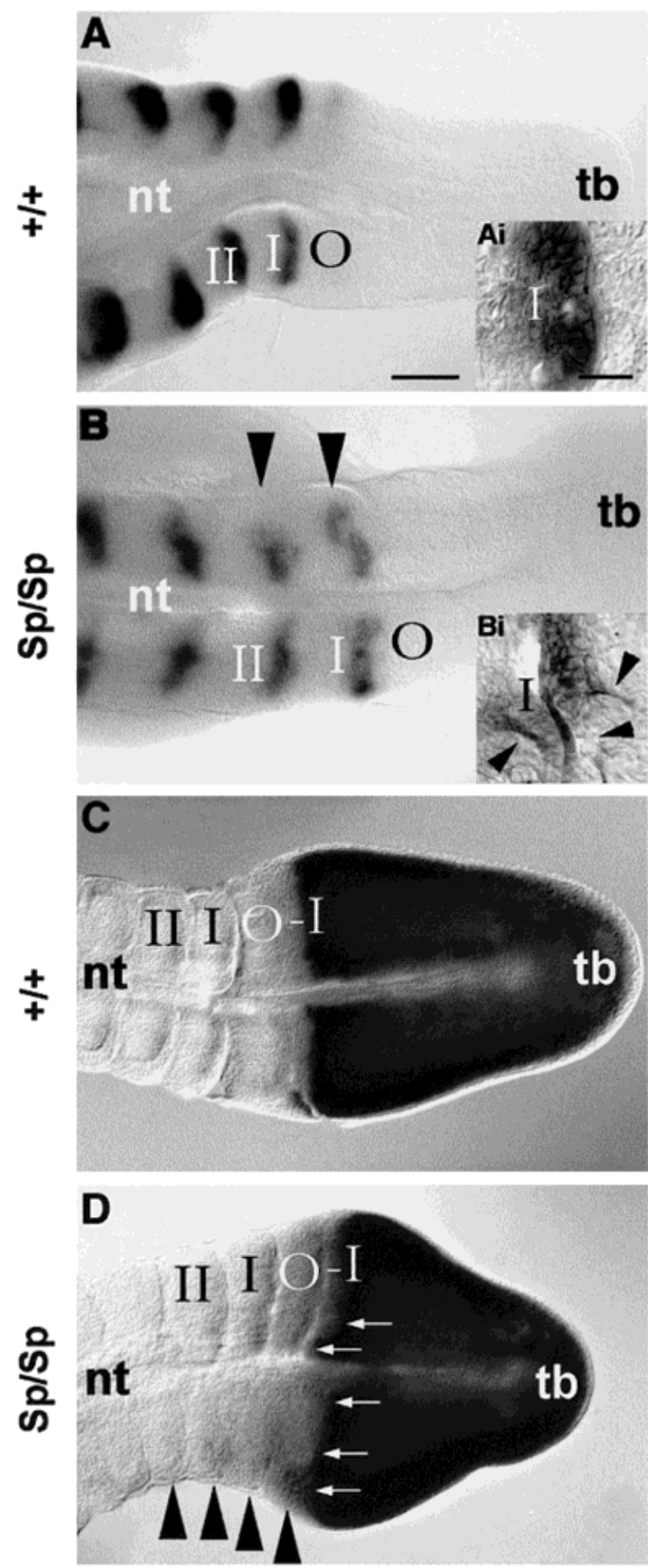

DII1 disturbed, and the rudiments of the spinal ganglia were displaced. Finally, fused signals were evident already in the youngest sclerotomes to express Pax1. Thus, the Splotch vertebral column malformations result from disorganised, early sclerotomes.

\section{Indirect Versus Direct Causes for the Splotch Vertebral Column Phenotypes}

Because the sclerotome is devoid of Pax 3 expression, Pax3-positive tissues have to be taken into account to explain the Splotch sclerotomal phenotypes. First, Pax3-deprived dorsal neural tube and neural crest cells may indirectly disturb the organisation of the sclerotome: Splotch mice are neural crest deficient, their dorsal neural tube is disorganised, even where neural closure proceeds (Russel, 1947; Auerbach, 1954; Tremblay et al., 1995; reviewed by Chalepakis et al., 1993). The dorsal neural tube provides critical signals for spinous process formation (Monsoro-Burq et al., 1996) that may be absent in Splotch. Likewise, the Splotch neural crest deficiency that leads to compromised dorsal root ganglia particularly in the lumbosacral region (Auerbach, 1954; Tremblay et al., 1995; reviewed by Chalepakis et al., 1993) may allow sclerotomal reorganisation, thereby leading to ectopic Pax1 up-regulation in the rostral sclerotome halves, to the formation of ectopic cartilage condensations and to misconnection and fusion of neural arches, as suggested by Hall, (1977). However, the sclerotome is generally believed to dictate the segregation of the spinal nerves rather receiving patterning signals from the emigrating neural crest cells (reviewed by Christ and Ordahl, 1995; Gossler and Hrabě de Angelis, 1998). Moreover, the Splotch neural crest deficiency seems not to be intrinsic to the mutant neural crest cells at all as these cells are motile in a wild-type environment (Serbedzija and McMahon, 1997) but fail to enter the Splotch sclerotome where the chondroitin sulphate proteoglycan Versican is erroneously up-regulated (Henderson et al., 1997). Finally, sclerotome induction and the formation of ventral vertebral column elements, namely vertebral bod-

Fig. 7. Expression of Uncx4.1 and DII1 during epithelial somite formation in wild-type $(A, C)$ and Splotch (B,D) embryos. A-D: Dorsal views on tail tips, inserts (Ai,Bi) $15 \mu \mathrm{m}$ frontal sections of somite $\mathrm{I}$, rostral is to the left in all. A,B: Uncx4.1 expression, day post coitum (dpc) 10.5. A, Ai: In the wild-type, the recently formed somites express the gene in clearly demarcated, regularly spaced stripes in the caudal somite halves. B, Bi: In Splotch, irregular Uncx4.1 signals indicate perturbed rostrocaudal somite pattern and defective somitic boundaries already at the level of the most recently formed somites (arrowheads). C,D: DIl1 expression, dpc 12.0. C: In the wild-type, DII1 signals are confined to the caudal margin of the recently formed somites as well as the epithelialising somite No. 0 . In the next somite to form (No. I), DII1 expression is down-regulated in the prospective rostral half. D: In Splotch, somites fail to fully segregate, with irregular or fused signals for DII1 (arrowheads). Note that somites on the left and right side are out of register. Defects can be traced back to the second somite to form (No. I), where the down-regulation of DII1 proceeds along an irregular front line (arrows). Abbreviations as before. Scale bar $=100 \mu \mathrm{m}$ in A (applies to A-D), $30 \mu \mathrm{m}$ in Ai (applies to Ai,Bi). 
ies and intervertebral discs, depend on notochord and floor plate rather than the dorsal neural tube (reviewed by Christ and Ordahl, 1995; Gossler and Hrabě de Angelis, 1998). Thus, although neural tube/ neural crest deficiencies may contribute to the malformation of the neural arches, they are unlikely to cause the full picture of vertebral malformations seen in Splotch.

As a second possibility, Splotch vertebral column phenotypes may be evoked by the Pax3-deficient dermomyotomes, which are truncated, disorganised, and fused, and deliver disorganised myotomes only (reviewed by Dietrich, 1999). Fate mapping studies in the chick proposed the lateral dermomyotome as source of the distal ribs (Kato and Aoyama, 1998), suggesting that the defective dermomyotomes in Splotch may directly result in truncated expression domains of Tenascin $C$ and Scleraxis in the mesenchymal rib anlagen, and in subsequent rib fusion (Henderson et al., 1999). Recently, the lateral sclerotome has been suggested to provide the rib precursors (Huang et al., 2000), and a more indirect mode of dermomyotome/myotome - sclerotome interaction has been proposed, based on the rib defects in mice deficient for the myotomal gene Myf5 (Braun et al., 1992) and on the up-regulated Versican expression in the lateral aspect of the Splotch sclerotome, beneath the dermomyotome/myotome complex (Henderson et al., 1997). Moreover, as Pax3-deficient dermomyotomes show enhanced apoptosis (Borycki et al., 1999), they may facilitate the expansion of the sclerotome even ventromedially and, as a consequence, the formation of ectopic cartilages. However, there is no indication that the Splotch sclerotomes are hypertrophied, i.e., the dorsoventral dimensions of the (medial) sclerotome, the dorsoventral dimensions of Pax1 signals, and the dorsoventral perimeter of vertebral bodies are unaltered (Henderson et al., 1997, 1999; Tremblay et al., 1998; this study). We directly addressed this question with a Drosophila-type compensation experiments, where we crossed Splotch mice hypothetically harbouring oversized sclerotomes with Pax1/undulated extensive mutants that posses underdeveloped sclerotomal condensations and lack lumbosacral vertebral bodies (Wallin et al., 1994; Dietrich and Gruss, 1995). Rather than compensating each other, the phenotypes of double homozygotes were additive, and the reduced $u n^{e x}$ sclerotomes did not cure the formation of ectopic or fused vertebral cartilages typical for Splotch. Finally, in the light of the recent Myf5 knockout, the idea of a defective myotome inhibiting the formation of ribs had to be revised (Kaul et al., 2000). Thus, although cross-talk between sclerotome, myotome, and dermomyotome cannot be ruled out and may contribute to the development of lateral vertebral components, Splotch vertebral column defects are likely to arise from deficiencies within the sclerotome.
Sclerotomal Disorders in Splotch Can Be Traced Back to the Initial Phase of Pax3 Expression in Segmenting Paraxial Mesoderm

As Pax3 is down-regulated in the developing sclerotome, any intrinsic sclerotomal deficiencies in Splotch must have been inherited from earlier defects in the paraxial mesoderm. Importantly, Pax3 is expressed at the rostral end of the unsegmented paraxial mesoderm, during mesoderm condensation, and during the formation of epithelial somites, thus, at the right time and place to act in the genetic cascades controlling mesoderm segmentation or somitogenesis (Goulding et al., 1994; Williams and Ordahl, 1994; this study). Consistent with a role for Pax3 during these processes, the Splotch vertebral column phenotype resembles the phenotypes of the segmentation/somitogenesis mutants pudgy and rachiterata (see Mouse Locus Catalogue, Jackson Laboratory; Kusumi et al., 1998) in that all three mutants show irregular or fused vertebral column elements at random distribution. In addition, they harbour disorganised and fused dermomyotomes (reviewed by Dietrich, 1999), myotomes (S. Dietrich, unpublished observations) and sclerotomes (this study). Moreover, rostrocaudal somite pattern and somitic boundaries are perturbed in Splotch. Most importantly, recently formed, epithelial somites as well as the next two somites to develop are affected as monitored by the disturbed expression pattern of Uncx4.1, EphA4 (Sek1), and Dll1, coinciding with the first phase of Pax3 expression in the paraxial mesoderm (summarised in Fig. 8). This finding strongly suggests that the vertebral column phenotypes seen in Splotch relate to improperly segregated somites, resulting from a defective organisation of the condensing paraxial mesoderm.

\section{Possible Role for Pax3 in the Formation of Epithelial Somites}

In Drosophila, the Pax3 homologue paired is a pairrule gene, involved in the regulation of segmentation (Nüsslein-Volhard and Wieschaus, 1980). Thus, it is possible that in vertebrates, the function of paired/ Pax3 genes in subdividing the embryo has been conserved. However, segmentation of vertebrates and (long germ band) insects is distinct (reviewed in Pourquié, 1999). Moreover, circumstantial evidence suggests that Pax3 is involved in somite epithelialisation rather than mesoderm segmentation: first, the periodic expression of Lunatic fringe in the unsegmented paraxial mesoderm is unaltered in Splotch, suggesting that segmentation per se proceeds normally. Second, timing and regulation of the early Pax3 expression is similar to Paraxis, a crucial regulator for somite epithelialisation (Fan and Tessier-Lavigne, 1994; Burgess et al., 1996; Dietrich et al., 1997; Šošić et al., 1997). Third, Pax3 and Paraxis expression are interdependent, as expression of Pax3 is reduced in Paraxis mutants (Burgess et al., 1996), whereas Paraxis expression is disor- 
Fig. 8. Schematic representation of murine Pax3 expression and onset of somitogenesis phenotypes in Splotch. Pax3 expression commences in the unsegmented paraxial mesoderm at the levels of somite -I, encompassing the entire dorsoventral perimeter. Markers such as Uncx4.1, EphA4, and DI/1 highlight somite segregation and rostrocaudal somite pattern at the levels of the recently formed somite (No. I), the somite undergoing epithelialisation (No. 0) and the next somite to epithelialise (No. I). Expression of these markers is perturbed in Splotch (arrowheads).

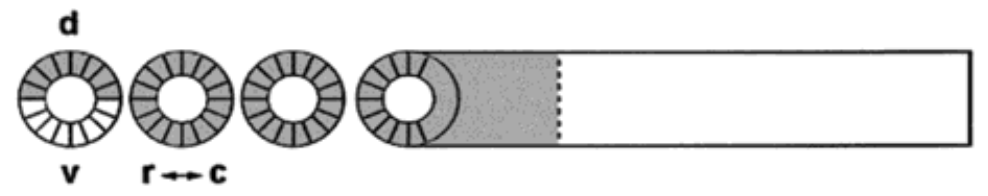

Pax3

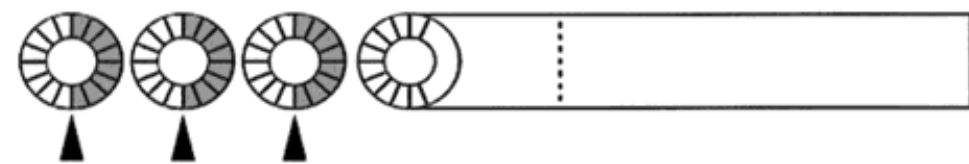

Uncx 4.1

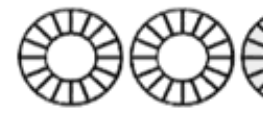

sing

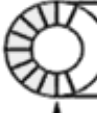

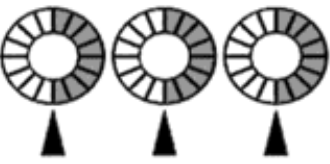

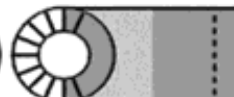

DII1

EphA4

$\frac{\mathrm{III} \text { II I }}{\text { somites }} \frac{\mathrm{O}}{\text { unsegmented }}$

ganised and subsequently lost in Splotch (Henderson et al., 1999), with both mutants showing the same disturbance of rostrocaudal somite pattern (Johnson et al., 2001; this study). Fourth, ectopic Pax3 signals in the paraxial mesoderm colocalise with ectopic epithelia (Dietrich et al., 1997, 1998). And finally, in the neural tube Pax3 is involved in regulating cell adhesion, as cells in the neural tube of Splotch are more loosely packed and the profile of NCAM isoforms is altered (Morris and O'Shea, 1983; Moase and Trasler, 1991; reviewed by Chalepakis et al., 1993). Thus, in the paraxial mesoderm, Pax3 may similarly regulate cell adhesion, thereby controlling compaction and epithelialisation of the paraxial mesoderm. Unlike Paraxis, however, the function of Pax3 for mesoderm epithelialisation may partly be rescued by the Pax3 paralogue Pax7, which shows a similar expression pattern in the unsegmented paraxial mesoderm, is up-regulated in Splotch, and in the dermomyotome, can partially compensate for the loss of Pax3 (Jostes et al., 1991; Goulding et al., 1994; Borycki et al., 1999; R. Mootosami and S. Dietrich, unpublished observations). In summary, although the direct interaction of the genes of question has yet to be established, we propose that in the segmenting paraxial mesoderm of amniotes, Pax3, downstream and in a feedback loop with Paraxis, and probably assisted by Pax7, acts in the formation of epithelial somites.

\section{EXPERIMENTAL PROCEDURES}

Mice

Wild-type control animals were obtained from NMRI $x$ B6D2 matings. The parental lines stem from the Zentralinstitut für Versuchstierzucht, Hannover, Germany. Splotch ( $S p), S p^{d}, S p 2 H$, pudgy, and rachiterata heterozygotes were obtained from the Jackson Laboratory (see also Mouse Locus Catalogue, Jackson Laboratory). Splotch mice were outcrossed with C57Bl6. Homozygotes and compound heterozygotes were obtained from heterozygous matings and identified on the basis of neural tube and muscular phenotypes as described by Tremblay et al. (1998). pudgy mutants were outcrossed with NMRI to increase viability. Homozygotes were obtained from $p u / p u \times p u / p u$ or from $p u /$ $p u \times p u /+$ matings. rachiterata mutants were outcrossed with C57Bl6. Subsequently, homozygous lines were established. Undulated extensive mice were kindly provided by J.L. Cruickshank, Leeds, UK. The mutants were outcrossed with C57B16, to subsequently establish homozygous lines as described by Dietrich and Gruss (1995). To generate $S p-u n^{e x}$ double mutants, we mated $S p /+$ with $u n^{e x} / u n^{e x}$. Animals with belly spots, indicating the $S p$ mutation, were backcrossed to $u n^{e x} / u n^{e x}$. We obtained offspring with kinky tails and belly spots at the expected ratio, indicating that Spun $^{e x} /+u n^{e x}$ are viable. These animals were mated 
inter se to obtain double homozygotes, which develop until dpc 13.5. All mice were mated overnight, taking $12 \mathrm{am}$ of the day of the vaginal plug as day 0.5. post coitum (dpc).

\section{Skeletal Preparations}

Skeletal preparations were performed according to Kessel et al. (1990). To photograph the vertebral column ventrally, the viscera were removed.

\section{Molecular Markers}

To detect the expression pattern of $\operatorname{Pax} 3$, we used the probes described by Goulding et al. (1991, 1994). The Pax1 expression pattern was determined by using the SspI-AluI probe described by Dietrich and Gruss (1995). For the Uncx4.1 staining, the probe described by Mansouri et al. (1997) was used. The EphA4 probe, kindly provided by P. Charnay, Paris, and D. Wilkinson, London, corresponds to the probe described by Nieto et al. (1992). The Dll1 probe was kindly provided by A. Gossler, Hannover, and corresponds to the probe described by Bettenhausen et al. (1995). Finally, we gratefully received the Lunatic fringe probe described by Johnston et al. (1997) from T. Vogt, Princeton.

\section{In Situ Hybridisation and Sectioning}

Whole-mount in situ hybridisation was performed as described by Dietrich et al. (1997). To indicate the progress of somite formation, we used the numbering system introduced by Ordahl and Christ (1995), modified by Pourquié (1999). After the staining reaction, embryos were embedded in gelatine for sectioning on a Pelco Vibratome. For cryosectioning, embryos were embedded in sucrose/gelatine before mounting them in Tissue-Tek O.C.T. compound.

\section{Photography}

Skeletons and whole-mounted embryos were photographed on a Zeiss Stemi SV8 binocular microscope and a Leica MZFLIII stereomicroscope. Details of whole-mounted embryos and sections were photographed on a Zeiss Axiophot microscope by using Nomarski optics.

\section{ACKNOWLEDGMENTS}

We thank P. Charnay and D. Wilkinson for the EphA4 probe, A. Gossler for the Dll1 probe, and T. Vogt for the Lunatic fringe probe. We also thank J.L. Cruickshank for providing a breading pair of $u n^{e x}$ mice, and the Jackson Laboratory for Splotch, pudgy, and rachiterata mice. We are grateful to C. Müller and J. Krull for excellent technical assistance.

\section{REFERENCES}

Auerbach R. 1954. Analysis of the developmental effects of a lethal mutation in the house mouse. J Exp Zool 127:305-329.

Aulehla A, Johnson RL. 1999. Dynamic expression of lunatic fringe suggests a link between notch signalling and an autonomous cellular oscillator driving somite segmentation. Dev Biol 207:49-61.
Barnes GL, Alexander PG, Hsu CW, Mariani BD, Tuan RS. 1997. Cloning and characterization of chicken Paraxis: a regulator of paraxial mesoderm development and somite formation. Dev Biol 189:95-111.

Bettenhausen B, Hrabě de Angelis M, Simon D, Guénet J-L, Gossler A. 1995. Transient and restricted expression during mouse embryogenesis of Dll1, a murine gene closely related to Drosophila Delta. Development 121:2407-2418.

Borycki A-G, Li J, Jin F, Emerson CP. 1999. Pax3 functions in cell survival and in Pax7 regulation. Development 126:1665-1674.

Braun T, Rudnicki MA, Arnold H-H, Jaenisch R. 1992. Targeted inactivation of the muscle regulatory gene $M y f 5$ results in abnormal rib development and perinatal death. Cell 71:369-382.

Burgess R, Rawis A, Brown D, Bradley A, Olson EN. 1996. Requirement of the paraxis gene for somite formation and musculoskeletal patterning. Nature 384:570-573.

Chalepakis G, Stoykova A, Wijnholds J, Tremblay P, Gruss P. 1993. Pax: gene regulators in the developing nervous system. J Neurobiol 24:1367-1384.

Christ B, Ordahl CP. 1995. Early stages of chick somite development. Anat Embryol (Berl) 191:381-396.

Del Barco Barrantes I, Elia AJ, Wünsch K, Hrabě de Angelis M, Mak TW, Rossant J, Conlon RA, Gossler A, de la Pompa JL. 1999. Interaction between Notch signalling and Lunatic fringe during somite boundary formation in the mouse. Curr Biol 9:470-480.

Deutsch U, Dressler GR, Gruss P. 1988. Pax-1, a member of a paired box homologous gene family, is expressed in segmenting structures during development. Cell 53:617-625.

Dietrich S. 1999. Regulation of hypaxial muscle development. Cell Tissue Res 296:175-182.

Dietrich S, Gruss P. 1995. undulated phenotypes suggest a role of Pax1 for the development of vertebral and extravertebral structures. Dev Biol 167:529-548.

Dietrich S, Schubert FR, Lumsden A. 1997. Control of dorsoventral pattern in the chick paraxial mesoderm. Development 124:38953908.

Dietrich S, Schubert FR, Healy C, Sharpe PT, Lumsden A. 1998. Specification of the hypaxial musculature. Development 125:22352249.

Durbin L, Brennan C, Shiomi K, Cooke J, Barrios A, Shanmugalingam S, Guthrie B, Lindberg R, Holder N. 1998. Eph signaling is required for segmentation and differentiation of the somites. Genes Dev 12:3096-3109.

Fan CM, Tessier-Lavigne M. 1994. Patterning of mammalian somites by surface ectoderm and notochord: evidence for sclerotome induction by a hedgehog homolog. Cell 79:1175-1186.

Forsberg H, Crozet F, Brown NA. 1998. Waves of mouse Lunatic fringe expression, in four-hour cycles at two-hour intervals, precede somite boundary formation. Curr Biol 8:1027-1030.

Gossler A, Hrabě de Angelis M. 1998. Somitogenesis. Curr Topics Dev Biol 38:225-287.

Goulding MD, Chalapakis G, Deutsch U, Erselius JR, Gruss P. 1991. Pax-3, a novel murine DNA binding protein expressed during early neurogenesis. EMBO J 10:1135-1147.

Goulding MD, Lumsden A, Paquette AJ. 1994. Regulation of Pax-3 expression in the dermomyotome and its role in muscle development. Development 120:957-971.

Grüneberg H. 1963. The pathology of development. Oxford: Blackwell Scientific.

Hall BK. 1977. Chondrogenesis of the somitic mesoderm. Adv Anat Embryol Exp Morphol 22:253-264.

Henderson DJ, Ybot-Gonzales P, Copp AJ. 1997. Over-expression of the chondroitin sulphate proteoglycan versican is associated with defective neural crest migration in the Pax 3 mutant mouse (Splotch). Mech Dev 69:39-51.

Henderson DJ, Conway SJ, Copp AJ. 1999. Rib truncations and fusions in the $\mathrm{Sp}^{2 \mathrm{H}}$ mouse reveal a role for Pax3 in specification of the ventro-lateral and posterior parts of the somite. Dev Biol 209:143158.

Huang R, Zhi Q, Schmidt C, Wilting J, Brand-Saberi B, Christ B. 2000. Sclerotomal origin of the ribs. Development 127:527-532. 
Jiang YJ, Aerne BL, Smithers L, Haddon C, Ish-Horowicz D, Lewis J. 2000. Notch signalling and the synchronisation of the somite segmentation clock. Nature 408:475-478.

Johnson J, Rhee J, Parsons SM, Brown D, Olson EN, Rawls A. 2001. The anterior/posterior polarity of somites is disrupted in Paraxisdeficient mice. Dev Biol 229:176-187.

Johnston SH, Rauskolb C, Wilson R, Prabhakaran B, Irvine KD, Vogt TF. 1997. A family of mammalian Fringe genes implicated in boundary determination and the Notch pathway. Development 124: $2245-2254$.

Jostes B, Walther C, Gruss P. 1991. The murine paired box gene, Pax7, is expressed specifically during the development of the nervous and muscular system. Mech Dev 33:27-37.

Kato N, Aoyama H. 1998. Dermomyotomal origin of the ribs as revealed by extirpation and transplantation experiments in chick and quail embryos. Development 125:3437-3443.

Kaul A, Köster M, Neuhaus H, Braun T. 2000. Myf 5 revisited: loss of early myotome formation does not lead to a rib phenotype in homozygous Myf5 mutant mice. Cell 102:17-19.

Kessel M, Balling R, Gruss P. 1990. Variations of cervical vertebrae after expression of a Hox-11 transgene in mice. Cell 61:301-308.

Kusumi K, Sun ES, Kerrebrock AW, Bronson RT, Chi D-C, Bulotsky MS, Spencer JB, Birren BW, Frankel WN, Lander ES. 1998. The mouse pudgy mutation disrupts Delta homologue Dll3 and initiation of early somite boundaries. Nat Genet 19:274-277.

Larsen WJ. 1993. Human embryology. New York: Churchill Livingstone.

Mansouri A, Yokota Y, Wehr R, Copeland NG, Jenkins NA, Gruss P. 1997. Paired-related murine homeobox gene expressed in the developing sclerotome, kidney, and nervous system. Dev Dyn 210:5365.

McGrew MJ, Dale JK, Fraboulet S Pourquié O. 1998. The Lunatic fringe gene is target of the molecular clock linked to segmentation in avian embryos. Curr Biol 8:979-982.

Moase CE, Trasler DG. 1991. N-CAM alterations in Splotch neural tube defect mouse embryos. Development 113:1049-1058.

Moloney DJ, Panin VM, Johnston SH, Chen J, Shao L, Wilson R, Wang Y, Stanley P, Irvine KD, Haltiwanger RS, Vogt TF. 2000. Fringe is a glycosyltransferase that modifies Notch. Nature 406: $369-375$.

Monsoro-Burq A-H, Duprez D, Watanabe Y, Bontoux M, Vincent C, Brickell P, Le Douarin N. 1996. The role of bone morphogenetic proteins in vertebral development. Development 122:3607-3616.

Morris GL, O'Shea KS. 1983. Anomalies of neuroepithelial cell associations in the Splotch mutant embryo. Dev Brain Res 9:408-410.

Neidhardt LM, Kispert A, Hermann BG. 1997. A mouse gene of the paired-related homeobox class expressed in the caudal somite compartment and the developing vertebral column, kidney and nervous system. Dev Genes Evol 207:330-339.
Nieto MA, Gilardi-Hebenstreit P, Charnay P, Wilkinson DG. 1992. A receptor protein tyrosine kinase implicated in the segmental patterning of hindbrain and mesoderm. Development 116:1137-1150.

Nüsslein-Volhard C, Wieschaus E. 1980. Mutations affecting segment number and polarity in Drosophila. Nature 287:795-801.

Palmeirim I, Dubrulle J, Henrique D, Ish-Horowicz D, Pourquié O. 1998. Uncoupling segmentation and somitogenesis in the chick presomitic mesoderm. Dev Genet 23:77-85.

Pourquié O. 1999. Notch around the clock. Curr Opin Genet Dev 9:559-565.

Russell WL. 1947. Splotch, a new mutation in the house mouse, Mus musculus. Genetics 32:102.

Serbedzija G, McMahon AP. 1997. Analysis of neural crest cell migration in Splotch mice using a neural crest-specific LacZ reporter. Dev Biol 185:139-147.

Sošić D, Brand-Saberi B, Schmidt C, Christ B, Olson EN. 1997. Regulation of paraxis expression and somite formation by ectodermand neural tube-derived signals. Dev Biol 185:229-243.

Töndury G, Theiler K. 1990. Entwicklungsgeschichte und Fehlbildungen der Wirbelsäule. Stuttgart: Hippokrates Verlag.

Tremblay P, Kessel M, Gruss P. 1995. A transgenic neuroanatomical marker identifies cranial neural crest deficiencies associated with the Pax3 mutant Splotch. Dev Biol 171:317-329.

Tremblay P, Dietrich S, Meriskay M, Schubert FR, Li Z, Paulin D. 1998. A crucial role for Pax3 in the development of the hypaxial musculature and the long-range migration of muscle precursors. Dev Biol 203:49-61.

Van Eeden FJM, Granato M, Schach U, Brand M, Furutani-Seiki M, Haffter P, Hammerschmidt M, Heisenberg C-P, Jiang Y-J, Kelsh RN, Odenthal J, Warga RM, Nüsslein-Volhard C. 1996. Mutations affecting somite formation and patterning in the zebrafish, Danio rerio. Development 123:153-164.

Van Eeden FJ, Holley SA, Haffter P, Nüsslein-Volhard C. 1998. Zebrafish segmentation and pair-rule patterning. Dev Genet 23:6576.

Van Straaten HW, Copp AJ. 2001. Curly tail, a 50-year history of the mouse spina bifida model. Anat Embryol (Berl) 203:225-237.

Wallin J, Koseki H, Wilting J, Ebensperger C, Christ B, Balling R. 1994. The role of Pax-1 in the development of the axial skeleton. Development 120:1109-1121.

Williams BA, Ordahl CP. 1994. Pax-3 expression in segmental mesoderm marks early stages in myogenic cell specification. Development 120:785-796.

Wilting J, Kurz H, Brand-Saberi B, Steching G, Yang YX, Hasselhorn H-M, Epperlein H-H, Christ B. 1994. Kinetics and differentiation of somite cells forming the vertebral column: studies on human and chick embryos. Anat Embryol (Berl) 190:573-581. 